\title{
El reto de diagnosticar la enfermedad de Whipple
}

\section{Whipple's Disease and its challenging diagnosis}

Filipa Amado, Petro Tavares, Carla Falçao, Alcina Ponte

Servicio de Medicina Interna. Centro Hospitalario de Leiria (CHL), Portugal

\section{ABSTRACT}

Whipple's Disease is a bacterial disease with particular involvement of small intestine, caused by the bacillus Tropheryma whipplei. Because it is rare and has nonspecific clinical manifestations, the diagnostic is usually late. Has good response to antibiotic therapy but can be fatal if not treated. It is necessary to monitor its clinical course for several years due to high risk of relapse. Authors describe the case of a female patient with intermittent diarrhea and abdominal pain for several years admitted with transient global amnesia, developed prominent gastrointestinal symptoms, and definitive diagnosis was only made by histology of duodenal biopsy fragments.

Keywords: Whipple Disease; Tropheryma whipplei; macrophages PAS-positive; antibiotics.

\section{INTRODUCTION}

Whipple's Disease (WD) is a rare, systemic infectious bacterial disease caused by the gram-positive bacillus Tropheryma whipplei ${ }^{1}$. Usually occurs in the gastrointestinal system, particularly in small intestine causing malabsorption, buy may affect any part of the body. It is characterized by a prolonged phase of unspecific symptoms resulting in delayed or missed diagnosis ${ }^{2}$. The prognosis is good if properly and early treated showing good response to antibiotic therapy. However, relapse is common so it is necessary close followup for several years ${ }^{2,3}$.

\section{CASE REPORT}

A 52-year-old caucasian woman presented to the emergency department with sudden amnesia for events related to that day. The patient's past medical history included intermittent diarrhea and abdominal pain for several years of unknown etiology, treated with messalazine and chlordiazepoxide + clinidium bromide. She reported no arthralgia, fever or any other systemic manifestation. Physical examination only revealed temporal and spatial disorientation and memory loss for recent events. The laboratory tests were normal as so as electrocardiogram, chest $X$-ray and computerized tomography (CT) of the brain. Admitted to the Internal Medicine department with the suspicion of transient ischemic accident (TIA), presenting resolution of symptoms within the first 48 hours. Brain magnetic resonance imaging (MRI) showed bilateral symmetrical, diffuse areas on the hippocampal heads suggesting areas of restriction compatible with transient global amnesia (Fig. 1). Transthoracic echocardiogram and neck vessel Doppler echocardiography showed no changes. Five days later, she developed nausea and vomiting, frequent and watery dejections at times containing mucus. Laboratory tests revealed significant increase in transaminases (AST 152 UI/L, ALAT 638 UI/L), GGT /796 UI/L), ALP (846 $\mathrm{UI} / \mathrm{L})$ and LDH (256 U/L). Abdominal CT revealed no changes. Upper endoscopy and colonoscopy were performed showing no changes. She started methylprednisolone and messala-

Fig.1.

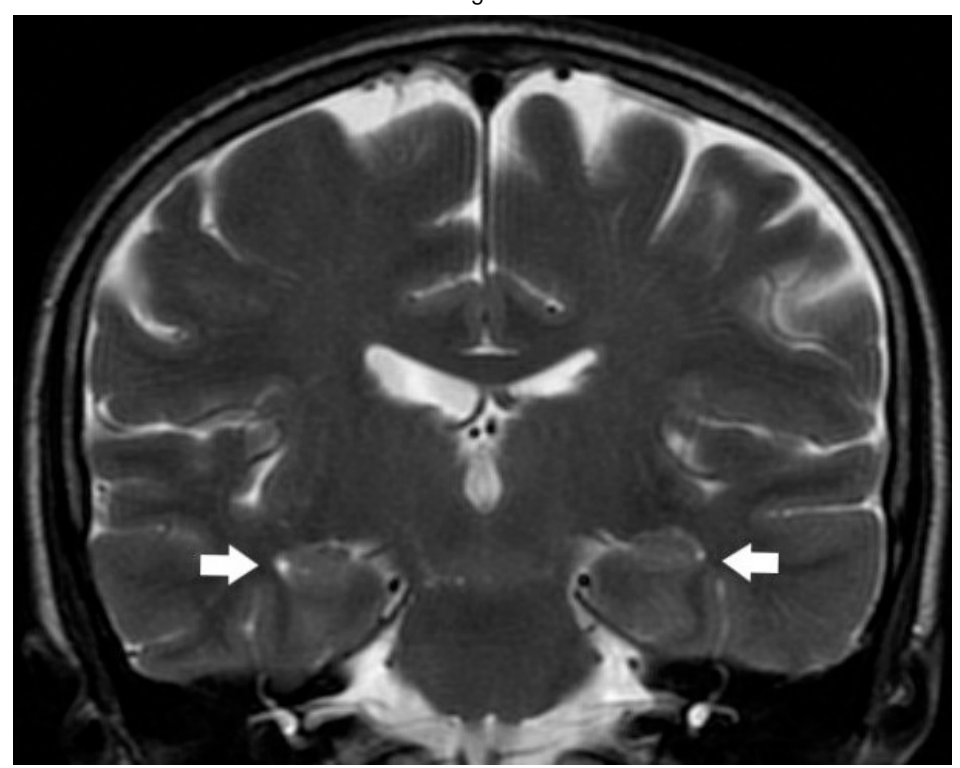


Fig.2-3.

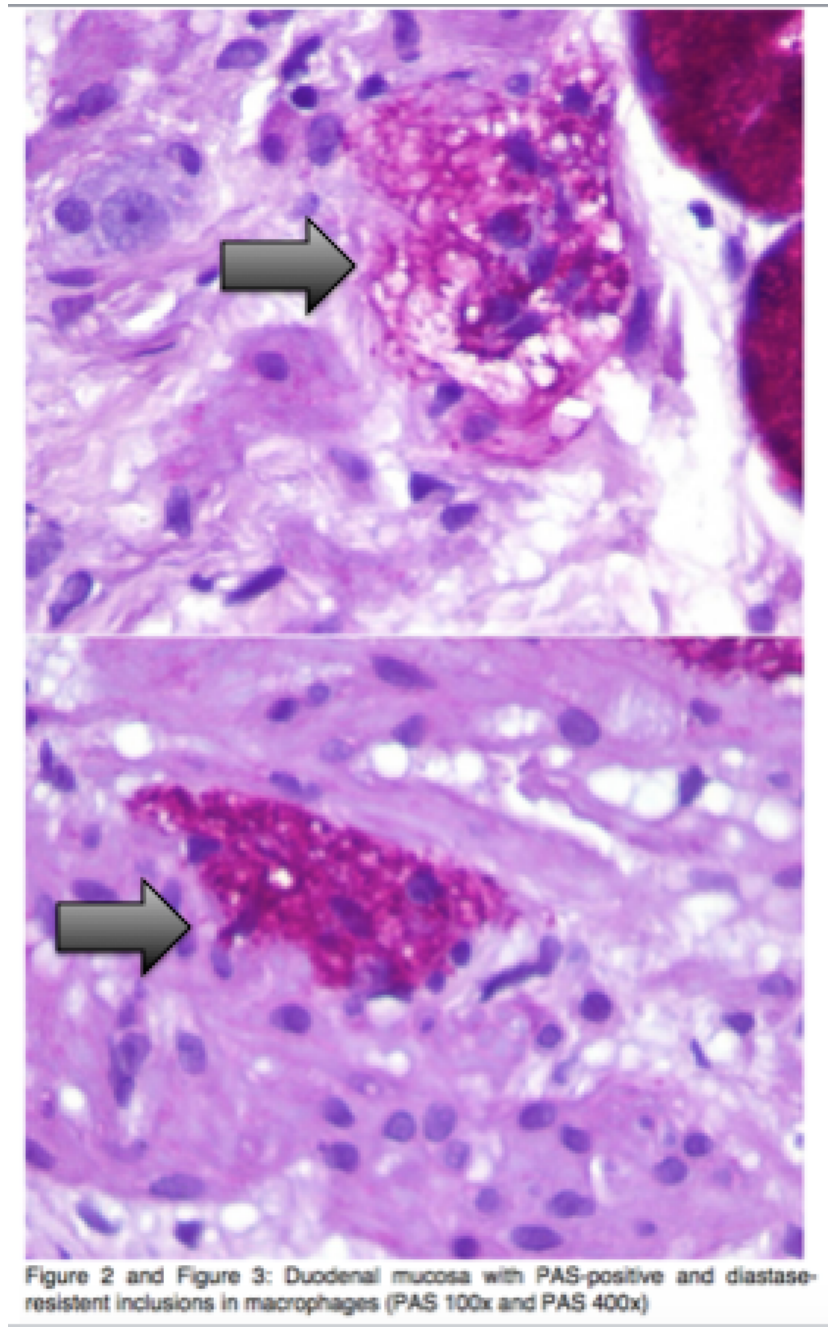

zine for a possible inflammatory bowel disease but remained with vomiting and diarrhea. Histological examination of multiple duodenal biopsies showed multiple PAS (periodic acid Schiff)-positive and diastase-resistant inclusions in foam macrophages, consistent with WD (Fig. 2 and 3). Polymerase chain reaction (PCR) was requested for Tropheryma whipplei but due to laboratory technical problems was not performed. Therefore, the diagnosis of WD with neurological involvement was made and $2 \mathrm{~g}$ daily ceftriaxone was administered intravenously for 2 weeks, and thereafter trimethoprim-sulfamethoxazole (160-800 mg) twice daily was started, but due to allergic reaction to this antibiotic, it was replaced by doxycycline twice daily. Disappearance of both vomiting and diarrhea occurred after seventeen days of treatment, but significant weight loss was observed. Four months after she was totally asymptomatic and has recovered $6 \mathrm{Kg}$ of weight. One-year oral therapy with doxycycline was performed. The patient continues to be asymptomatic after four years of follow-up.

\section{DISCUSSION}

Whipple's Disease is a chronic disease firstly described in 1907 by George Hoyt Whipple as "intestinal lipodystro- phy"4. Caused by the actinomycete Tropheryma whipplei, a gram-positive, PAS-positive obligate intracellular bacillus, suspected as aetiologic agent by PCR in 1992. Is natural habitat is not yet totally known although it has been found in soil, sewage and human feces, but also in samples of saliva, gastric juice and duodenal biopsies from individuals without the disease. There is evidence for a genetic predisposition to Tropheryma whipplei infection, and like other chronic infections, the immune system significantly contributes to the development of clinical manifestations. The overall incidence is 1-6:10.000.000 persons, with an estimated 12 new cases per year. It is more common in Caucasian men, with an average age of 50 years at the time of diagnosis. Clinical presentation includes intestinal, particularly affecting small intestine, and extra-intestinal symptoms, with consequent misdiagnosis ${ }^{5}$. The most frequent manifestations are diarrhea and weight loss leading to malabsorption syndrome, arthralgias and/or arthritis, abdominal pain, fever and peripheral and abdominal lymphadenopathies (mesenteric and retroperitoneal). Diarrhea is often steatorrhea but can also be watery. Arthralgias are nondestructive, migratory and involve large joints. They often precede diagnosis by several years. At least one-third of patients will have cardiac involvement but only few are symptomatic. Central nervous system (CNS) involvement may occur in the absence of gastrointestinal manifestations ${ }^{6}$. The most frequent neurological signs are cognitive changes, eye movement disorders, movement, language and hypothalamic disorders, seizures, ataxia and amnesia. Rare manifestations are skin hyperpigmentations, uveitis and chorioretinitis. Our patient had diarrhea and abdominal pain for several years which could already be related to the development of the disease. She had no history of arthralgias and presented with CNS involvement with global amnesia and hippocampal changes. As exemplified in our case, the condition requires a high index of suspicion to diagnose. Analytical changes are nonspecific and include microcytic and hypochromic anemia, lymphocytopenia, thrombocytosis, elevated sedimentation rate. Imaging changes are nonspecific too. Endoscopically the most characteristic is the thickening of the mucosa. The diagnosis is based on duodenal or jejunal biopsy with identification of PAS-positive macrophages resistant to diastasis ${ }^{7}$. Electron microscopy has contributed to the detection of the bacillus since 1961 but is currently reserved for cases where PCR and/or histology are doubtful. PCR in WD has high sensitivity and specificity and is especially useful in cases with atypical manifestations and/or when histological diagnosis cannot be confirmed $^{8}$. The differential diagnosis is vast and includes infections by Mycobacterium avium complex, Rhodococcus, Bacillus cereus, in which PAS-positive foamy macrophages can also be found, tuberculosis, inflammatory bowel disease with arthropathy, gastrointestinal vasculitis, celiac disease, sarcoidosis, among others. In the case of our patient, given gastrointestinal symptoms not explained by other etiologies and the involvement of the CNS, it was thought to be WD and 
the diagnosis was finally clarified by duodenal biopsy. Antibiotic therapy is the mainstay of medical treatment and usually leads to improvement of clinical conditions within two or three weeks. Given the frequent involvement of the CNS and being the most frequent site of relapse, use of antibiotics that penetrate the blood-brain barrier is recommended ${ }^{9}$. Treatment should be given for a period of no less than one year. The standard initial treatment is either ceftriaxone or penicilin $\mathrm{G}$ both for 14 days, followed by maintenance therapy with trimethoprim-sulfamethoxazole twice daily for one year. Even with proper treatment, the disease may relapse after several years, so follow-up should be continued for at least 10 years. With prompt diagnosis and treatment, the prognosis is WD is good. Most symptoms disappear in about a month and PCR becomes negative within a few weeks. Our patient had clinical improvement in the first weeks after starting therapy as is characteristic of the disease. Monitoring with duodenal biopsies at 6-12 months of treatment is desirable or using PCR to monitor disease during and after treatment, as negative PCR excludes disease recurrence because of its negative predictive value of $100 \%$. Despite advances in knowledge of WD, it will remain a diagnostic challenge.

\section{REFERENCES}

1. Carneiro AC, Lima P, Barbosa IP, Chaves FC. Doença de Whipple: um desafio diagnóstico. Acta Med Port. 2004; 17:481-486

2. Rocha HC, Marques Martins WR, Carvalho MR, Amaral LM. Manifestações clínicas e diagnóstico da doença de Whipple: relato de caso. Rev Med Minas Gerais. 2015; 25(2):296-300

3. Oliveira L, Gorjão R, Deus JR. Doença de Whipple. J Port Gastrenterol. 2010; 17:69-77

4. Dolmans RA, Boel CH, Lacle MM, Kustersa JG. Clinical Manifestations, Treatment, and Diagnosis of Tropheryma whipplei Infections. Clin Microbiol Rev. 2017;30(2):529-555

5. De Francesco V, Corsi F, Pennella A, Bellesia A, Fiorini G, Vaira D, Zullo A. Whipple's Disease: Case Report and Review of the Literature. J Gastrointestin Liver Dis. 2018; 27(3): 331-336

6. Crews NR, Cawcutt KA, Pritt BS, Patel R, Virk A. Diagnostic Approach for Classic Compared With Localized Whipple Disease. Open Forum Infect Dis. 2018; 5(7):0fy136.

7. Abreu P, Azevedo E, Lobo L, Moura CS, Pontes C. Doença de Whipple e sistema nervoso central. Acta Med Port. 2005; 18:199-208

8. Günther U, Moos V, Offenmüller G, Oelkers G, Heise W, Moter A, et al. Gastrointestinal diagnosis of classical Whipple disease: clinical, endoscopic, and histopathologic features in 191 patients. Medicina (Baltimore). 2015; 94:e714

9. Moter A, Janneck M, Wolters M, Iking-Konert C, Wiessner A, Loddenkemper C, et al. Potencial Role for Urine Polymerase Chain Reaction in the Diagnosis of Whipple's Disease. Clin Infect Dis. 2019; 68:1089 